\title{
CIRUGÍA CONSERVADORA DE RIÑÓN PARA TUMORES RENALES PEQUEÑOS, PAPEL DE LA RADIOFRECUENCIA
}

\author{
Alfredo Velascol, Octavio Castilloly2, Ivar Vidal', Rafael Sánchez-Salas', Rodrigo Campos', \\ Renato Cabello', Felipe Balbontin', Alejandro Majerson ${ }^{3}$ y Gilberto González³.
}

'Unidad de Enndourología y Laparoscopía Urológica, Clínica Santa María, Santiago, Chile.

2Departamento de Urología, Facultad de Medicina, Universidad de Chile, Santiago, Chile.

${ }^{3}$ Hospital Clínico Pontificia Universidad Católica de Chile.

Resumen.- OBJETIVO: La radiofrecuencia con asistencia laparoscópica es una opción de tratamiento minimamente invasivo para la conservación de parénquima renal, especialmente en pacientes con comorbilidad aumentada. Se presentan los resultados a corto plazo de los pacientes tratados por esta novedosa técnica.

MÉTODOS: Las lesiones renales menores de $4 \mathrm{~cm}$., sospechosas de malignidad o de metástasis a la TC o RNM son candidatas para radiofrecuencia. Bajo visión laparoscópica el tumor es identificado, realizándose una biopsia por punción percutánea. Según tamaño del tumor, se realiza un determinado número de punciones con aguja de radiofrecuencia, con el fin de lograr la

\section{CORRESPONDENCIA}

Octavio Castillo

Unidad de Endourología y

Laparoscopía Urológica

Clínica Santa María

Av. Apoquindo 3990, Of. 809

Las Condes. Santiago de Chile.

octaviocastillo@vtr.net

Trabajo recibido: 24 de marzo 2008 necrosis del tumor durante al menos 1 ciclo de radiofrecuencia. El seguimiento es realizado con RNM en el día post operatorio 1 y luego con TC o RNM al mes, 3,6 y 12 meses. La ausencia persistente de contraste - la necrosis vascular de la lesión es considerada una ablación satisfactoria sin recurrencia.

RESULTADOS: Doce pacientes, 2 por enfermedad metastásica y 10 por lesiones primarias ledad promedio 60.8 años), con una o más lesiones sospechosas de malignidad fueron tratadas con radiofrecuencia. EI ASA promedio fue de 2,4. El número de tumores tratados fue de 15 con un diámetro de $2,8 \mathrm{~cm}$. Se utilizaron un promedio de 2,5 punciones con aguja de radiofrecuencia. Los resultados de la biopsia fueron metástasis de $7^{\circ}$ tiroídeo: 1 paciente, metástasis de melanoma: 1 paciente y cáncer de células renales en 10 pacientes. La estadía hospitalaria promedio fue de 25.8 horas. No existieron complicaciones a corto plazo. Tiempo de seguimiento 8.8 meses. Hasta hoy no hay evidencias de recidiva en los controles imagenológicos.

CONCLUSIONES: La radiofrecuencia es efectiva en erradicar lesiones renales pequeñas, tanto primarias como metastásicas, siendo especialmente útil en pacientes con comorbilidad aumentada. A pesar de que no hay una cantidad suficiente de pacientes con seguimiento adecuado, esta tecnología es prometedora. El abordaje bajo visión laparoscópica contribuye a una biopsia efectiva, evitando diseminación y permite una radiofrecuencia más certera al constatar bajo visión directa la necrosis del tumor.

Palabras clave: Ablación por radiofrecuencia. Tumores renales. Cirugía minimamente invasiva. 
Summary.- OBJECTIVES: Laparoscopically assisted radiofrequency is a minimally invasive nephron-sparing treatment option for renal tumors, mainly in patients with high comorbidity. We present the short-term results of our series patients treated with this novel technique.

METHODS: Renal lesions smaller than $4 \mathrm{~cm}$, suspicious of malignancy or metastasis on CT scan or MRI are candidates for radiofrequency. Under laparoscopic vision the tumor is identified, and percutaneous biopsy is performed. Depending on the size of the tumor, a number of punctures with the radiofrequency needle are performed with the aim to achieve fumor necrosis during at least one cycle of radiofrequency. Follow-up is performed with MRI in the first postoperative day and then after CT scan or MRI at 1, 3, 6 and 12 months. The persistent absence of contrast or vascular necrosis of the lesion is considered a satisfactory ablation without recurrence.

RESULTS: 12 patients, two with metastasis and ten with primary lesions (mean age 60.8 years), with one or more lesions suspicious of malignancy underwent radiofrequency. Mean ASA was 2.4. 15 tumors were treated, with a mean diameter of $2.8 \mathrm{~cm}$. An average of 2.5 punctures was performed with the radiofrequency needle. Biopsy results showed: one case of thyroid cancer metastasis, one case of melanoma metastasis, and 10 cases of renal cell carcinoma. Mean hospital stay was 25.8 hours. There were not short-term complications. Follow-up time was 8.8 months. Today there is no evidence of recurrence in imaging tests.c,

CONCLUSIONS: Radiofrequency is effective eradicating small renal lesions, both primary and metastatic; it is especially useful in patients with high comorbidity. Despite the number of patients with adequate follow-up is not enough, the technology is promising. The approach under laparoscopic vision contributes to an effective biopsy, avoiding dissemination and enabling a more precise radiofrequency by direct vision control of tumor necrosis.

Keywords: Radiofrequency ablation. Renal tumours. Minimally invasive surgery.

\section{INTRODUCCIÓN}

Con el advenimiento y la diseminación de las modalidades de imagen abdominal ha habido un marcado incremento del número de las masas renales descubiertas en pacientes asintomáticos, que se estima en un $75 \%$. Las masas sólidas descubiertas incidentalmente, son generalmente pequeñas, solitarias, no están asociadas con metástasis y tal vez no se asocian a malignidad como aquellas descubiertas por síntomas derivados de la masa. De echo muchos han propuesto el seguimiento de estas lesiones en casos seleccionados $(1,2)$. En ocasiones estas masas son descubiertas en pacientes con co morbilidad significativa o en pacientes añosos, lo cuál crea un dilema para el clínico que debe balancear el riesgo de la lesión para la vida del paciente $\mathrm{v} / \mathrm{s}$ la morbimortalidad del tratamiento.

El estándar de oro para el carcinoma de células renales confinado al riñón ha sido la nefrectomía radical, procedimiento que puede ser exagerado considerado las características del tumor y las condiciones generales del enfermo. Durante las últimas 3 décadas la cirugía conservadora de riñón ha sido aceptada como una alternativa apropiada a la nefrectomía radical en pacientes seleccionados. Las ventajas de la cirugía conservadora de riñón incluyen la preservación de función renal, estableciendo una reserva ante un eventual deterioro del riñón contralateral o la aparición de nuevos tumores. Por otro lado, cabe destacar que los tumores benignos son infrecuentes y que la diferenciación entre carcinoma renal y tumor benigno es dificultosa con las actuales técnicas de imágenes, lo cuál plantea cirugías conservadoras. Las desventajas de esta alternativa incluyen una falla en reconocer y tratar enfermedad multifocal, las recurrencias en el lecho resecado y la mayor morbilidad asociada a la nefrectomía parcial.

La nefrectomía parcial no está exenta de morbilidad, ya sea realizada por vía abierta o laparoscópica. Más aún desde que las masas renales son descubiertas más comúnmente en población añosa, que tiende a tener co morbilidad, los procedimientos

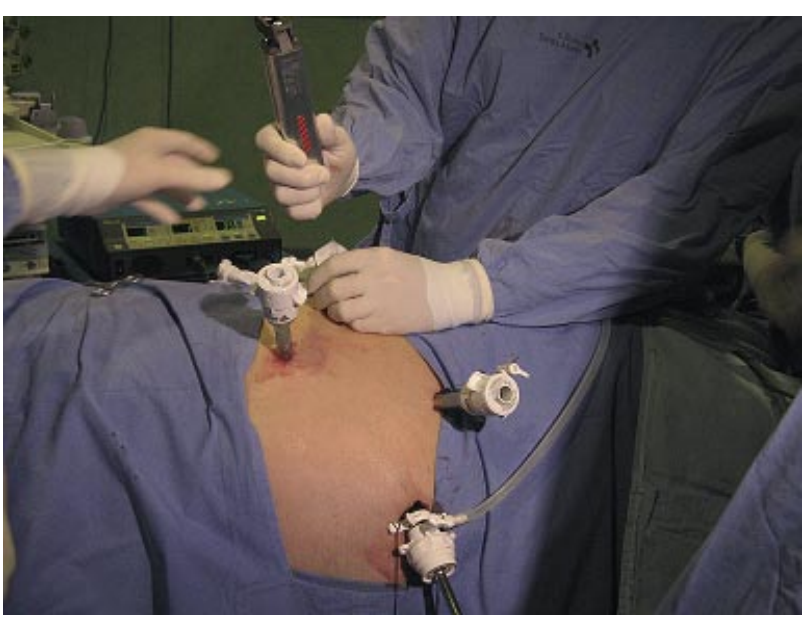

FIGURA 1. Biopsia por punción percutánea del tumor renal. 
quirúrgicos mayores en esta población se espera que estén asociados a una mayor morbilidad. Por lo tanto existe una necesidad de técnicas ablativas conservadoras de riñón, que puedan erradicar el tumor sin la morbilidad de una cirugía abierta, disminuyendo el sangrado, dolor post operatorio y con una corta estadía hospitalaria. La radiofrecuencia fue aprobada por la FDA en un principio para tumores hereditarios (Von Piel Lindau y Birdh Hodge Dubbe) y posteriormente para tumores esporádicos (2002). Hoy en día constituye una alternativa real de tratamiento, ya que permite tratar tumores pequeños, múltiples, bilaterales y hereditarios, lesiones metastásicas y tratar enfermos de alto riesgo quirúrgico, sin limitar el número de procedimientos en el caso de tumores bilaterales. Puede ser usada en forma percutánea y laparoscópica para la ablación de cánceres renales, existiendo ventajas y desventajas de cada una. Generalmente la vía percutánea presenta menos morbilidad, aunque es una técnica ciega y con mayor posibilidad de realizar el procedimiento sin certeza, es por esto que en ocasiones la vía laparoscópica es preferida, ya que permite aislar el tumor de estructuras vitales cuando ellas están adyacentes al tumor, permitiendo además una biopsia certera y radiofrecuencia más efectiva bajo visión directa del tumor.

Nuestra intención es presentar los resultados a corto plazo de los pacientes tratados por esta novedosa técnica.

\section{MATERIAL Y MÉTODOS}

Evaluación prospectiva de pacientes con lesiones renales exofíticas menores de $4 \mathrm{~cm}$., sospechosas de malignidad o de metástasis a la TC o RNM

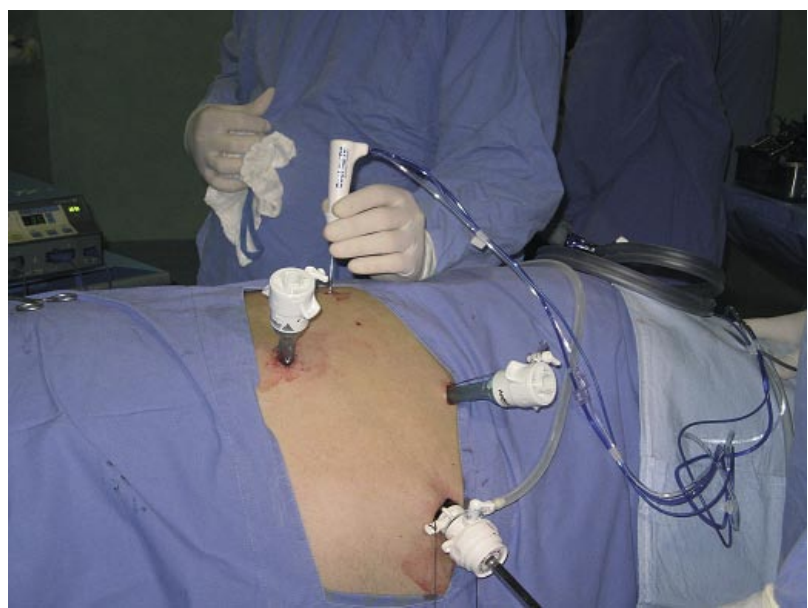

que fueron sometidas a ablación por radiofrecuencia, entre Abril de 2005 y diciembre de 2007.

\section{Mecanismo y técnica de radiofrecuencia}

En 1891, un físico francés llamado D' Arsanval (3), reportó el paso de una corriente alterna de radiofrecuencia a través del hígado para generar calor, pero sin causar excitación neuromuscular. Esta observación inspiró posteriormente el desarrollo del bisturí de Bovie, que fue usado ampliamente en los quirófanos por más de 70 años.

En 1990, McGahan y cols. (4) and Rossi y cols. (5), fueron los primeros en reportar el uso de RFA para el manejo de neoplasias hepáticas en modelos animales. Desde entonces osteomas osteoides y pequeños tumores hepáticos primarios y secundarios han sido tratados exitosamente con RFA. Básicamente los parámetros de RFA son similares para el hígado y riñón (6), La RFA puede ser utilizada por tecnología mono o bipolar, siendo la técnica monopolar más comúnmente usada. Cuando la técnica monopolar es realizada, la placa es puesta en el muslo del paciente. La placa actúa como un gran electrodo dispersivo que

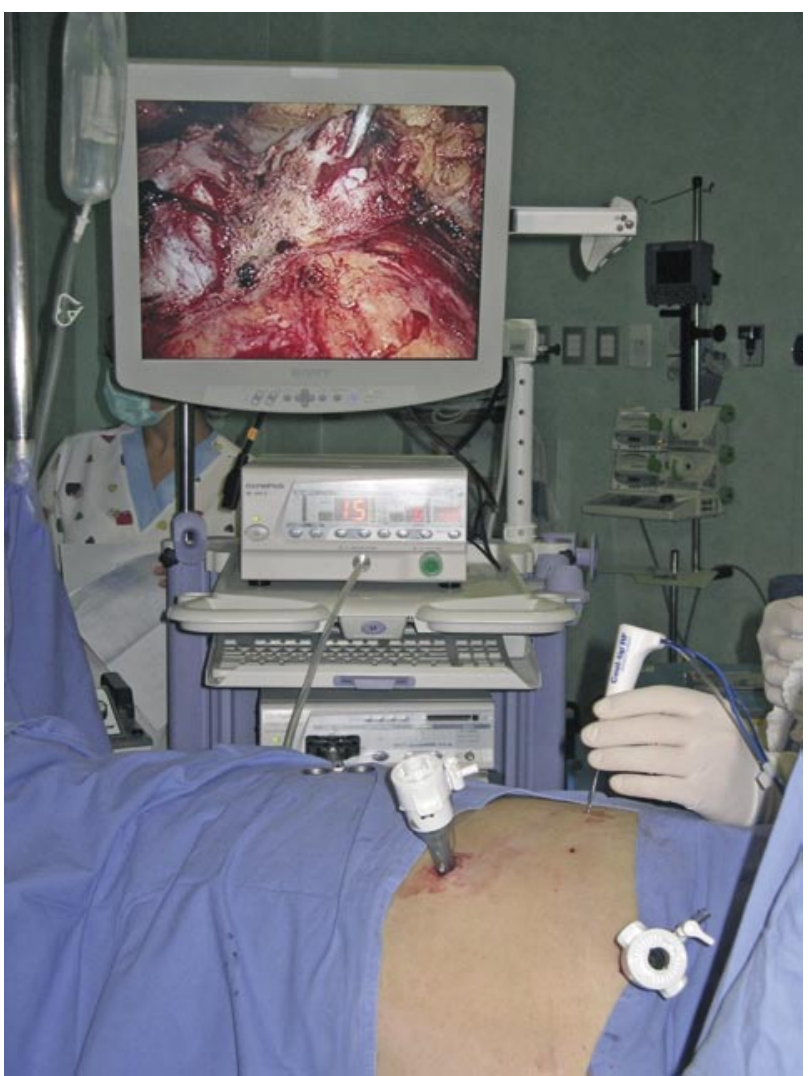

FIGURAS 2 y 3. Ablación por radiofrecuencia de tumor renal asistida por laparoscopia. 
permite que la corriente pase a través del paciente, el generador, activando el electrodo activo como un circuito. Cuando una corriente alterna de 460 - 500 $\mathrm{kHz}$ fluye desde el generador, entre los electrodos y la placa en el muslo, ocurre una agitación iónica en el tejido, siendo esto convertido por medio de la fricción en color. Esto resulta en una lesión termal focal en el tejido alrededor de la punta del electrodo activo $(7,8)$. Los generadores de RF actuales, pueden automáticamente ajustar el poder de salida para poder optimizar la energía depositada durante el tratamiento de ablación. Mientras mayor densidad de corriente rodeando la aguja del electrodo, más energía es depositada en el tejido, incrementando por lo tanto la cantidad de ablación (7). La homeostasis puede ser mantenida cuando la temperatura es elevada a aproximadamente $40{ }^{\circ} \mathrm{C}(8)$. Cuando la temperatura alcanza $46^{\circ} \mathrm{C}$ un daño celular irreversible ocurre en el tejido, pero no necrosis. Después que la temperatura alcanza $50-52^{\circ} \mathrm{C}$, sólo 4 a 6 minutos son necesarios para inducir un efecto citotóxico sobre la célula tumoral (9). Ocurre necrosis de coagulación y resulta en un daño termal irreversible a las células debido a la pérdida de la actividad enzimática citosólica y mitocondrial (7). Sobre $60^{\circ}$ la muerte celular es casi instantánea. Con la elevación de la temperatura, el diámetro de la necrosis de coagulación aumenta, pero varía en los diferentes tejidos dependiendo de la conducción y composición. A temperaturas sobre $105^{\circ} \mathrm{C}$, la ablación producirá ebullición, producción de gas y carbonización. El gas es un aislante que previene la transmisión de energía. Una clave para la ablación es la mantención de una temperatura entre $50-100^{\circ} \mathrm{C}$ en todo el volumen tumoral, sin producir vaporización de gas.

Existen múltiples sistemas de RF, pero todos constan básicamente de un generador eléctrico, un electrodo y una placa. El generador posee un detector que registra la resistencia e impedancia de los tejidos, de esta forma sólo destruye la zona del riñón donde hay tumor con gran precisión. Previo al procedimiento se puede tomar una biopsia por punción para su análisis patológico. Así también en caso de tumores profundos, estos pueden se ubicados con ecografía intraoperatoria de modo de destruir áreas precisas no detectables con cirugía habitual.

Las estrategias de sobrelapar los tratamientos de RF, varían con el tamaño de cada lesión. Estimando una injuria termal de $3 \mathrm{~cm}$. para cada tratamiento de ablación, un tumor de menos de $2 \mathrm{~cm}$. de diámetro puede ser tratado con uno o dos punciones. Las agujas de radiofrecuencia presentan diferentes diámetros, lo que permite tratar lesiones de diferente tamaño, existiendo un halo de seguridad con la generación de la radiofrecuencia.

\section{Técnica}

Bajo visión laparoscópica el tumor es identificado, realizándose una biopsia por punción percutánea directa de la zona del tumor, utilizándose una aguja de trucut para evitar la diseminación del tumor (Figura 1).

Dependiendo del tamaño del tumor, se realiza un determinado número de punciones con aguja de radiofrecuencia de diferentes diámetros según el tamaño del tumor, con el fin de lograr la necrosis del tumor durante al menos 1 ciclo de radiofrecuencia. Se utilizó un equipo de radiofrecuencia marca Tyco (Valleylab - Cool-tip ${ }^{\mathrm{TM}}$ RF Ablation System) (Figuras 2 y 3$)$.

El enfriamiento de la punta del electrodo se llevó a cabo utilizando solución salina enfriada. Una vez que la aguja fue instalada en la posición correcta, sesiones de tratamiento de hasta 12 minutos de duración fueron utilizadas, registrando la impedancia. El equipo fue ajustado para tiempo de radiofrecuencia según impedancia, es decir la radiofrecuencia actuó mientras existieran células que transmitieran. Cuando se registraba necrosis completa el equipo suspende la emisión de radiofrecuencia (Figura 4).

Durante el procedimiento, no es necesario realizar clampeo del pedículo renal, por lo que no hay isquemia caliente, a diferencia de la nefrectomía parcial abierta o laparoscópica, esto debido a que no hay sangrado.

La aguja dispone de un sensor que detecta la impedancia y determina la ausencia de vitalidad del tumor, permitiendo realizar ciclos de ablación más cortos.

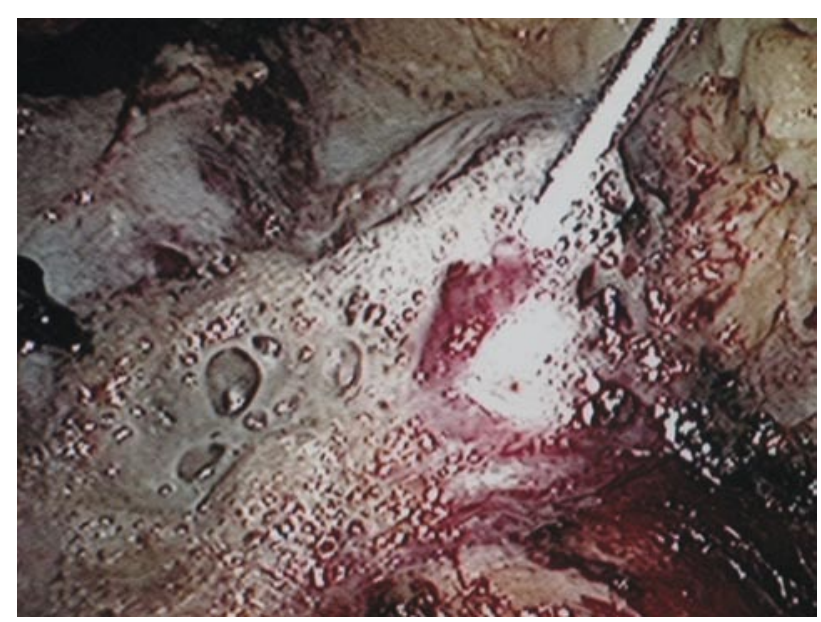

FIGURA 4. Necrosis por coagulación del tumor. 


\begin{tabular}{|c|c|c|c|c|c|c|c|c|c|c|c|c|c|}
\hline $\begin{array}{l}\text { o. } \\
\bar{\Xi} . \\
\underline{0} \\
\bar{O} .\end{array}$ & $\begin{array}{l}\text { 꼬․ } \\
\overline{3} \\
\text { 음. } \\
\text { ㄱ. }\end{array}$ & 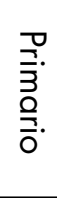 & $\begin{array}{l}\text { 꾸․ } \\
\overline{3} \\
\overline{3} \\
\text { 으․ } \\
\text { ․․․ }\end{array}$ & 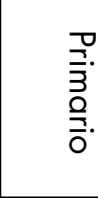 & $\begin{array}{l}\text { 모. } \\
\overline{3} . \\
\text { 을. } \\
\text { ․․․ }\end{array}$ & $\begin{array}{l}\text { 므. } \\
\overline{3} . \\
\text { 음. } \\
\text { o. }\end{array}$ & $\begin{array}{l}\text { 모․ } \\
\overline{3} \\
\text { 윽. } \\
\text { ․ }\end{array}$ & $\begin{array}{l}\text { 므. } \\
\overline{3} \\
\text { 음. } \\
\text { o. }\end{array}$ & $\begin{array}{l}\text { 모. } \\
\overline{3} . \\
\text { 음. } \\
\text { ․ }\end{array}$ & $\begin{array}{l}\text { 꼬․ } \\
\overline{3} . \\
\text { 음. } \\
\text { o. }\end{array}$ & $\begin{array}{l}3 \\
\frac{1}{0} \\
\frac{0}{0} \\
\frac{0}{0} \\
\frac{0}{0} \\
\frac{1}{2} \\
0\end{array}$ & $\begin{array}{l}3 \\
\frac{1}{0} \\
0 \\
\frac{0}{9} \\
\frac{0}{0} \\
\frac{0}{n} \\
0 \\
0\end{array}$ & $\begin{array}{l}\text { 독 } \\
\text { 옥 }\end{array}$ \\
\hline ০ & 2 & ga & ఏ & जे & $\stackrel{\infty}{\cup}$ & $\searrow$ & $\searrow$ & $\stackrel{U}{\Delta}$ & y̆ & a & $\omega$ & $\stackrel{\sim}{\circ}$ & $\frac{\pi}{2}$ \\
\hline$\equiv$ & $\equiv$ & $\equiv$ & $\equiv$ & $=$ & $=$ & $=$ & $=$ & $=$ & $\equiv$ & ₹ & $=$ & $=$ & $\frac{B}{5}$ \\
\hline 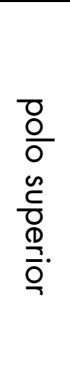 & 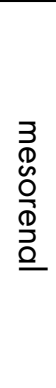 & 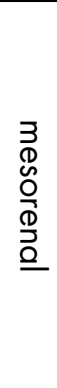 & 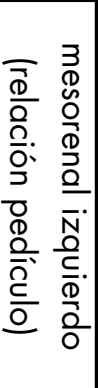 & 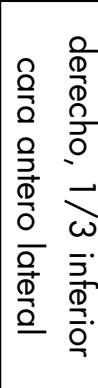 & 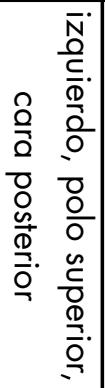 & 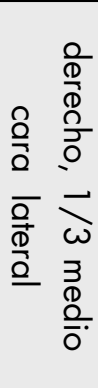 & 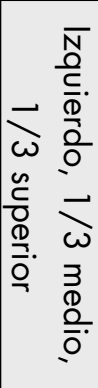 & 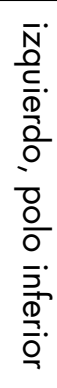 & 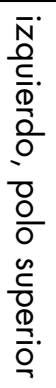 & 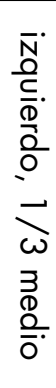 & 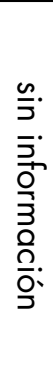 & 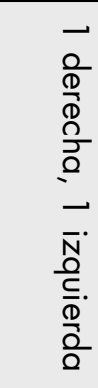 & $\begin{array}{l}\text { 둥. } \\
\text { 응 } \\
\text { 응: }\end{array}$ \\
\hline- & - & - & - & - & - & - & $N$ & - & - & - & - & $N$ & 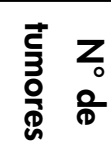 \\
\hline 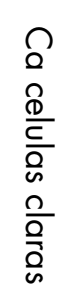 & 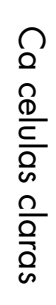 & $\begin{array}{l}\Omega \\
0 \\
\frac{0}{0} \\
\frac{c}{0} \\
0 \\
0 \\
\frac{0}{0} \\
\frac{0}{0}\end{array}$ & 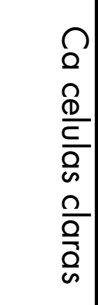 & $\begin{array}{l}\Omega \\
8 \\
\frac{\Phi}{0} \\
\frac{0}{0} \\
0 \\
0 \\
\frac{0}{0} \\
\frac{0}{0}\end{array}$ & $\begin{array}{l}\Omega \\
\Omega \\
\Omega \\
\frac{D}{C} \\
\frac{O}{0} \\
\omega \\
\frac{O}{0} \\
\frac{0}{3} \\
\omega\end{array}$ & 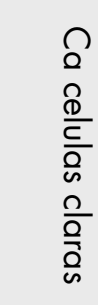 & 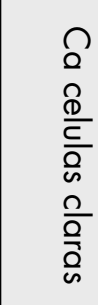 & 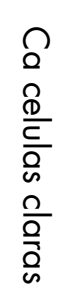 & $\begin{array}{l}\Omega \\
0 \\
\frac{0}{0} \\
\frac{c}{0} \\
0 \\
0 \\
\frac{0}{0} \\
\frac{0}{0} \\
0\end{array}$ & $\begin{array}{l}\Omega \\
0 \\
\frac{0}{0} \\
\frac{c}{0} \\
0 \\
0 \\
\frac{0}{0} \\
0 \\
0\end{array}$ & $\begin{array}{l}\frac{3}{10} \\
\frac{1}{0} \\
\frac{0}{0} \\
\frac{0}{3} \\
0\end{array}$ & 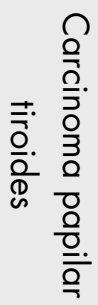 & 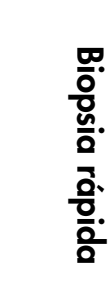 \\
\hline$N$ & $\stackrel{N}{\infty}$ & $\stackrel{N}{u}$ & $N$ & $\begin{array}{l}N \\
\dot{u}\end{array}$ & $\begin{array}{l}N \\
O\end{array}$ & $\begin{array}{l}\stackrel{N}{\alpha} \\
\alpha\end{array}$ & $\begin{array}{l}\omega \\
\dot{N} \\
\dot{\omega} \\
\dot{\omega}\end{array}$ & $\vec{i}$ & $N$ & $N$ & $N$ & $\begin{array}{l}\dot{1} \\
\dot{a}\end{array}$ & $\begin{array}{l}\text { 뭉: } \\
\frac{0}{3} \\
\frac{0}{3} \\
\frac{0}{3} \\
\underline{3}\end{array}$ \\
\hline$N$ & $\Delta$ & $\omega$ & $N$ & G & $\Delta$ & - & $N$ & $\omega$ & $\omega$ & $\omega$ & - & $\begin{array}{l}N \\
= \\
\stackrel{N}{D} \\
\stackrel{-}{-} \\
\bar{N} \\
\stackrel{0}{=}\end{array}$ & 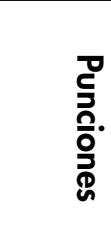 \\
\hline$\stackrel{N}{\Delta}$ & $\stackrel{N}{\Delta}$ & $\stackrel{N}{\Delta}$ & $\stackrel{N}{\Delta}$ & $\stackrel{N}{\Delta}$ & $\omega$ & $\stackrel{N}{\Delta}$ & $\stackrel{\Delta}{\Delta}$ & $\stackrel{\sim}{\Delta}$ & $\stackrel{\sim}{\Delta}$ & $\omega$ & $\stackrel{N}{\Delta}$ & $\stackrel{N}{\Delta}$ & 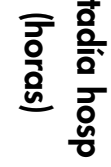 \\
\hline$N$ & $G$ & $a$ & $V$ & $V$ & $V$ & $\infty$ & $\infty$ & $\infty$ & $\infty$ & $\infty$ & O & $\underline{\sim}$ & 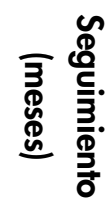 \\
\hline
\end{tabular}




\section{Seguimiento}

Es realizado con RNM en el día post operatorio 1 y luego con TC o RNM al mes, 3, 6 y 12 meses. La ausencia persistente de contraste o la necrosis vascular de la lesión es considerada una ablación satisfactoria sin recurrencia. No se realizó Re-biopsia.

\section{RESULTADOS}

Doce pacientes, 2 por enfermedad metastásica y 10 por lesiones primarias, dos de ellas Bosniak III o mas que en la biopsia intraoperatoria fueron informadas como adenocarcinoma (edad promedio 60.8 años), con una o más lesiones sospechosas de malignidad fueron tratadas con radiofrecuencia. El ASA promedio fue de 2,4. El número de tumores tratados fue de 15 con un diámetro promedio de $2.8 \mathrm{~cm}$ (1 - 3). Se utilizaron un promedio de 2,5 punciones con aguja de radiofrecuencia. Los resultados de la biopsia fueron metástasis de $1^{\circ}$ tiroídeo (metástasis bilateral): 1 paciente, metástasis de melanoma: 1 paciente y cáncer de células renales en 10 pacientes. La estadía hospitalaria promedio fue de 25.8 horas. El sangrado no fue cuantificable, no existiendo ningún paciente con sangrado evidente durante el procedimiento. No existieron complicaciones a corto plazo. Tiempo de seguimiento 8.8 meses. Hasta hoy no hay evidencias de recidiva en los controles imagenológicos. La tabla siguiente resume las características de los pacientes (Tabla I).

\section{DISCUSIÓN}

Este seguimiento a corto plazo de los pacientes con comprobación histológica de carcinoma renal o metástasis, tratados con ablación por radiofrecuencia, sugiere que en pacientes seleccionados esta modalidad de tratamiento es efectiva y probablemente equivalente en el control tumoral a la cirugía abierta. El procedimiento es bien tolerado, incluso en pacientes con comorbilidad elevada. En nuestra serie fue utilizado en pacientes con contraindicación de cirugía por riesgo alto y en un paciente que debía recibir terapia anticoagulante a las 24 horas de la cirugía, por lo que se contraindicaba el tratamiento convencional o laparoscópico, por el alto riesgo de sangrado post operatorio.

Son conocidas las ventajas de la cirugía laparoscópica, pero muchas veces conlleva una morbilidad importante, siendo la mas temida de ellas el sangrado, cuestión que con la radiofrecuencia es menor ya que no se han descrito como complicación importante. Finalmente hay algunos enfermos que presentan complicaciones mayores, como es el caso de uno de los pacientes de la serie, que es monorreno debido a una nefrectomía por cáncer previa, cuatro años atrás. Éste último presentaba un tumor meso renal izquierdo en íntima relación con la arteria y vena renal, lo cuál contraindicaba o hacía altamente riesgosa una nefrectomía parcial abierta o laparoscópica. A lo anterior se sumaba un clearence de creatinina de $33 \mathrm{ml} / \mathrm{min}$., por lo que el solo clampeo de la arteria, implicaba un deterioro de la función renal. Esto último no es necesario en la ARF, ya que el sangrado es mínimo y no hay riesgo de sangrado activo.

Existen diferentes técnicas para mejorar el resultado de la RF. La inyección intraparenquimatosa de compuestos que contienen fierro o solución salina antes y/o durante la RF, mejoraría la conducción del calor dentro del parénquima (10). De igual manera la utilización de energía pulsada ha demostrado ser efectiva al disminuir el calor y la cantidad de burbujas de gas. La irrigación continua de suero fisiológico en la pelvis renal durante el procedimiento mediante un catéter ureteral prevendría la necrosis y posterior aparición de fístulas en la vía urinaria. Por último la instilación percutánea de etanol, seguido a la terapia de RF, incrementa la extensión de la necrosis de coagulación y ha demostrado su efectividad en estudios a mediano plazo (11).

La combinación de cirugía laparoscópica y de equipos de radiofrecuencia de avanzada, permite que la ARF sea segura, con una tasa extremadamente baja de complicaciones mayores. Permite además visualizar el tumor, realizar una biopsia dirigida evitando la siembre tumoral o del trayecto, como también realizar una ablación dirigida y controlada. Esto último permite evitar la principal complicación descrita que es la recurrencia tumoral, secundaria a una ablación incompleta. Complicaciones severas derivadas de la RF, son poco comunes (Hemorragia, lesión del sistema colector, fístula urinaria, lesión de órganos adyacentes y lesiones cutáneas por el uso de la placa) y no existieron en nuestra serie. Otras complicaciones menores incluyen hematuria microscópica, dolor y reacciones anestésicas. La hematuria microscópica es común y no necesita tratamiento.

Actualmente la mejor indicación para ARF para el tratamiento de tumores renales, es una lesión exofítica, menor de $3 \mathrm{~cm}$. de diámetro mayor. El tamaño y la configuración de la lesión tratada, son los que influencian principalmente a la cantidad de energía administrada, impedancia del tejido, tiempo de ablación y área de superficie de los electrodos. A pesar de que es técnicamente imposible controlar las variables independientemente, nuestros resultados preliminares son aceptables y alentadores. 


\section{CONCLUSIONES}

La radiofrecuencia es efectiva en erradicar lesiones renales exofíticas pequeñas, tanto primarias como metastásicas.

A pesar de que no hay una cantidad suficiente de pacientes con seguimiento adecuado, esta tecnología es prometedora y tiene un rol significativo en el manejo de los tumores renales. Más aún los pacientes añosos y aquellos con comorbilidad significativa, tendrían menos complicaciones con la radiofrecuencia que con la resección quirúrgica.

El abordaje bajo visión laparoscópica contribuye a una biopsia efectiva, evitando diseminación y una radiofrecuencia más efectiva al constatar bajo visión la necrosis del tumor.

\section{BIBLIOGRAFÍA Y LECTURAS

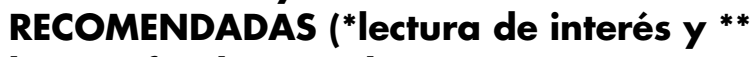 lectura fundamental)}

*1. Luciani L, Cestari R and Tallarigo C. Incidental renal cell carcinoma-age and stage characterization and clinical implications: study of 1092 patients (1982-1997). Urology 2000; 56: 58.

2. Pantuck A J, Zisman A, Rouch M K et al. Incidental renal tumors. Urology 2000; 56: 190.
3. D'Arsanval M A. Action physiologique des courants alternatifs. CR Soc Biol 1891; 43: 28386.

4. McGahan JP, Browing PD, Brock JM et al. Hepatic ablation using radiofrequency electrocautery. Invest Radiol 1990; 25: 267-70.

5. Rossi S, Fornari F, Pathies $\mathrm{C}$ et al. Thermal lesions induced by $480 \mathrm{kHz}$ localized current field in guinea pig and pig liver. Tumori 1996; 76:54-57.

*6. Zlotta AR, Wildshutz T, Raviv G et al. Radiofrequency interstitial tumor ablation (RITA) is a possible new modality for treatment of renal cancer: ex vivo and in vivo experience. J Endourol 1997; 11:251-58.

7. Goldberg SN, Gazelle GS, Compton CC et al. Treatment of intrahepatic malignancy with radiofrequency ablation. Radiologic- pathologic correlation. Cancer 2000; 88: 2452-63.

**8. Goldberg SN, Gazelle GS, Mueller PR. Thermal ablation therapy for focal malignancy: a unified approach to underlying principles, techniques, and diagnostic imaging guidance. AJR Am J Roentgenol 2000; 174: 323-31.

9. Goldberg SN, Gazelle GS, Compton CC et al. Radio-frequency tissue ablation of VX2 tumor nodules in the rabbit lung. Acad Radiol 1996; 3 : 929-35.

10. Gillams AR. Thermal ablation of liver metastases. Abdom Imaging 2001; 26:361-68.

11. Fotiadis NI, Sabharwal T, Morales JP et al. Combined percutaneous radiofrequency ablation and ethanol injection of renal tumours: Midterm results. Eur Urol 2007; 52: 777-84. 